\title{
Measurement of the single top production with the CMS detector
}

\author{
Andrey Popov ${ }^{* \dagger}$ \\ SINP MSU \\ E-mail: aa.popovephysics.msu.ru
}

\begin{abstract}
The single top quark production is measured in both $t$ and tW channels in pp collision data at $\sqrt{s}=7 \mathrm{TeV}$, recorded at LHC with the CMS detector. The $t$ channel cross section was measured with the integrated luminosity $36 \mathrm{pb}^{-1}$ with the two complementary analyses. The first one exploits the specific angular properties of the signal with data-driven background estimates. The second analysis takes the advantage of the excellent agreement of the CMS simulation with the data and probes for the whole compatibility of the candidate events with the signal hypothesis using the boosted decision trees. The measurement in $\mathrm{tW}$ channel is performed with the integrated luminosity $2.1 \mathrm{fb}^{-1}$. The analysis' strategy is based on counting the events that pass the dedicated selection, and the impact of the major backgrounds is estimated from the data. The measured cross sections in both $t$ and $\mathrm{tW}$ channels are compatible with the Standard Model expectations.
\end{abstract}

The XXth International Workshop High Energy Physics and Quantum Field Theory

September 24-October 1, 2011

Sochi Russia

\footnotetext{
* Speaker.

†n behalf of CMS collaboration
} 


\section{Introduction}

The theory of electroweak interactions predicts three different production mechanisms for the single top quark in pp collisions in addition to the much more abundant pair production. They can be classified depending of the virtuality of W boson: $s$ channel (Fig. 1a), W-associated or tW channel (Fig. 1b), and $t$ channel (Fig. 1c). The $t$ channel is the dominant in pp collisions at $7 \mathrm{TeV}$ and $\mathrm{tW}$ channel is the second one. Depending on the analysis one of them is treated as the signal whereas the other two are included in the background. Only electronic and muonic registration channels are considered for analysis.

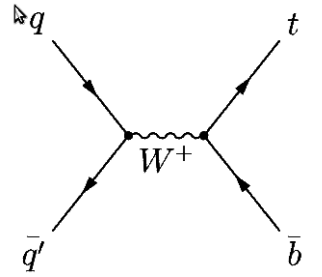

(a)

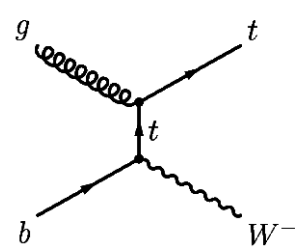

(b)
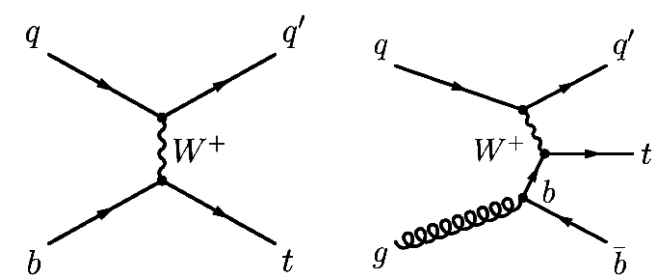

(c)

Figure 1: Example Feynman diagrams for the electroweak top quark production.

The $t$ channel cross section is measured with the integrated luminosity $35.9 \pm 1.4 \mathrm{pb}^{-1}$ recorded with the CMS detector up to November 2010. Two complementary analyses are performed [1]. The first one makes use of the specific angular distributions of the signal, the second one is based on boosted decision trees and probes the candidate events for compatibility with the electroweak theory expectation. In the following text these analyses are referred to as 2D and BDT respectively. Finally they are combined to obtain a single $t$ channel cross section measurement.

The $\mathrm{tW}$ channel analysis [2] exploits the integrated luminosity $2.1 \mathrm{fb}^{-1}$ recorded up to August 2011. The cross-section is measured with a counting experiment. The most important backgrounds are estimated with data-driven procedures.

\section{Analysis in $t$ Channel}

\subsection{Event Selection}

The signal event signature is exactly one isolated charged lepton (muon or electron since $\tau$ leptons are not considered in the analysis explicitly), central b-jet, and forward light-quark jet. It agrees with the event selection described below. Here and in the following text some of the quality requirements for the physical objects (like jet's composition, conversion rejection, minimum number of hits in the tracker, etc.) are omitted and can be found in Refs. [1,2].

An event is required to pass a trigger based on the presence of a muon or an electron. It must contain exactly one tight muon (electron) with transverse momentum (energy) $p_{T}>20 \mathrm{GeV} / c$ $\left(E_{T}>30 \mathrm{GeV}\right)$, pseudorapidity $|\eta|<2.1(|\eta|<2.5)$, and relative isolation $I<0.05(I<0.1)$ in $2 \mathrm{D}$ analysis or $I<0.1$ for both lepton flavours in BDT. The relative isolation is defined as the sum of transverse momentum of all the tracks and the transverse energies of the calorimeter deposits 
within the cone of size $\Delta R=\sqrt{\Delta \eta^{2}+\Delta \phi^{2}}<0.3$ around the lepton (excluding the lepton itself) divided by the lepton's transverse momentum. The event is rejected if it contains an additional loose muon (electron) with $p_{T}>10 \mathrm{GeV} / c\left(E_{T}>15 \mathrm{GeV}\right),|\eta|<2.5$, and $I<0.2$.

The event must contain exactly two jets with $p_{T}>30 \mathrm{GeV} / c$ and $|\eta|<5$. Exactly one of them must be tagged as a b-jet passing the tight working point of the tagging algorithm. The tagging algorithm is based on the significance of the 3D impact parameter of tracks associated to the jet. For 2D analysis only the second jet is required to fail the loose working point of the algorithm.

The QCD multijet background is reduced demanding $M_{T}>40(50) \mathrm{GeV} / c^{2}$ in muon (electron) channel. The transverse mass is defined as

$$
M_{T}=\sqrt{\left(p_{T}(l)+p_{T}(v)\right)^{2}-\left(p_{x}(l)+p_{x}(v)\right)^{2}-\left(p_{y}(l)+p_{y}(v)\right)^{2}} .
$$

\subsection{Top Quark Reconstruction}

The precise knowledge of $\mathrm{W}$ boson mass is used to provide a kinematic constraint on the 4-momentum of the top quark. This results in a quadratic equation for the longitudinal neutrino momentum $p_{z}(v)$. If two real solutions are present, the one with smallest $\left|p_{z}(v)\right|$ is chosen (it is correct in $60.3 \%$ of the cases). If there are no real solutions, $\not_{T}$ is modified to give $M_{T}=M_{\mathrm{W}}$.

\subsection{Data-Driven Background Estimation}

The analysis probes a very specific kinematic phase space region populated by the tails of distribution of QCD multijet events ("QCD" in the following text), hence this background is estimated from data. The shapes of QCD templates used below are taken from an anti-isolated region in data which is constructed by inverting the relative isolation requirement in the event selection. This region is by far dominated by QCD. The amount of QCD in the selection is estimated with a fit to transverse mass histogram:

$$
F\left(M_{T}\right)=a \cdot S\left(M_{T}\right)+b \cdot B\left(M_{T}\right),
$$

where the template $S\left(M_{T}\right)$ for sum of all processes but QCD is taken from the simulation, and the QCD template $B\left(M_{T}\right)$ is extracted from data.

\subsection{Cross-Section Measurement}

\subsubsection{D Analysis}

Due to V-A structure of the weak interaction, the produced top quark is almost $100 \%$ polarized with respect to the spin axis. This distinctive feature is propagated to $\cos \theta_{\mathrm{lj}}^{*}-\operatorname{cosine}$ of the angle, in the reconstructed top quark rest frame, between the decay lepton and the direction of the untagged jet (Fig. 2a). The second characteristic distribution used in the $2 \mathrm{D}$ analysis is the pseudorapidity of the light-flavour jet $\eta_{\mathrm{lj}}$, which demonstrates a notable non-centrality for the signal (Fig. 2b).

The cross-section is determined with the $2 \mathrm{D}$ fit to $\cos \theta_{\mathrm{lj}}^{*}-\mathrm{vs}-\eta_{\mathrm{lj}}$ distribution. The shapes for QCD and $\mathrm{W}+$ light partons are taken from data, all the other processes are taken from the simulation. During the fit the amount of signal and the overall amount of the background float unconstrained, whereas the relative composition of the background is fixed. 


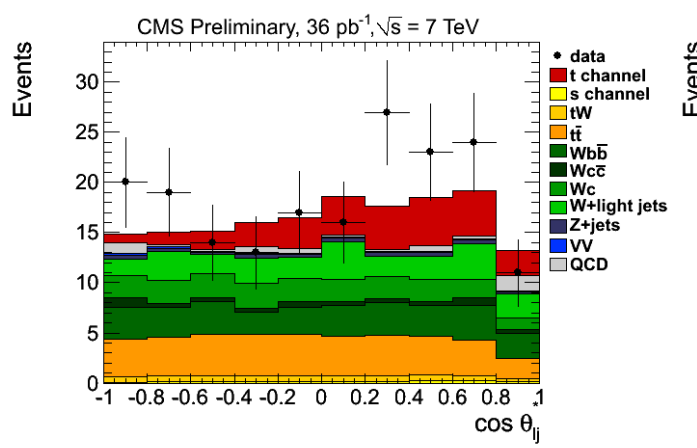

(a) $\cos \theta_{1 \mathrm{j}}^{*}$

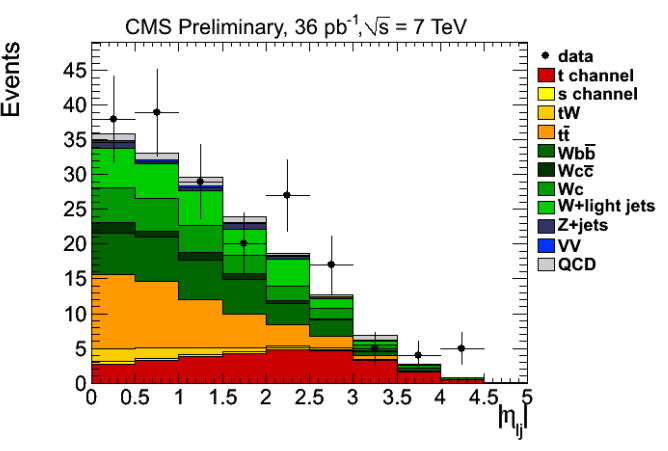

(b) $\left|\eta_{\mathrm{lj}}\right|$

Figure 2: Distributions in the variables for 2D analysis after the dedicated event selection. Muon and electron channels summed.

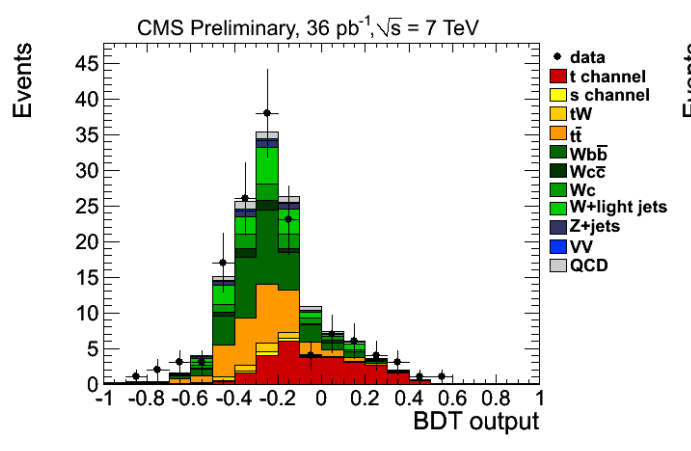

(a) Muon channel

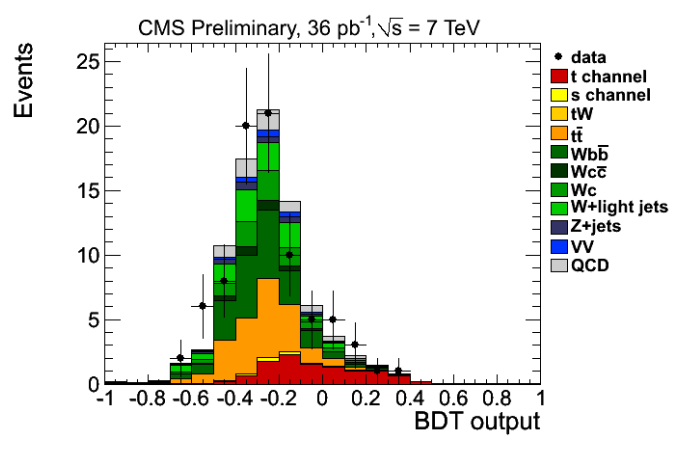

(b) Electron channel

Figure 3: BDT output in the signal region. The background are scaled to the posterior medians.

\subsubsection{BDT Analysis}

The BDT analysis uses the boosted decision trees to combine several observables into a single 1D powerful classifier. 37 variables are exploited in total, they can be split into several categories, each reflecting specific event properties: kinematics and individual properties of the leptons and jets, correlations between these objects, properties of the derived objects (W boson, top quark), angular distributions between the original and derived objects, global event characteristics (sphericity, scalar sum of transverse energy, etc.). First the most sensitive variables are: the lepton's transverse momentum, $\hat{s}$ (the mass of the system of $\mathrm{W}$ and the two jets), dijet transverse momentum, transverse momentum of the most b-tagged jet, and the reconstructed top mass.

The cross section is extracted from a binned likelihood fit to the BDT discriminator output (Fig. 3) within the full Bayesian approach. The QCD background is estimated from data and simulation used for the rest processes.

\subsection{Systematic Uncertainties}

The limited volume of the article does not make it possible to provide all the details of the 


\begin{tabular}{|c||c|c|}
\hline Channel & 2D analysis & BDT analysis \\
\hline \hline$\mu$ & $104.1 \pm 42.3_{-28.0}^{+24.8} \pm 4.2 \mathrm{pb}$ & $90.4 \pm 35.2_{-19.7}^{+16.5} \pm 3.6 \mathrm{pb}$ \\
\hline$e$ & $154.2 \pm 56.0_{-46.6}^{+40.6} \pm 6.2 \mathrm{pb}$ & $59.2 \pm 35.1_{-13.7}^{+13.1} \pm 3.6 \mathrm{pb}$ \\
\hline$\mu+e$ & $124.2 \pm 33.8_{-33.9}^{+30.0} \pm 5.0 \mathrm{pb}$ & $78.7 \pm 25.4_{-14.6}^{+13.2} \pm 3.1 \mathrm{pb}$ \\
\hline
\end{tabular}

Table 1: The $t$ channel cross section measurements by channel and by the analysis. The uncertainties are: statistical, systematic, and luminosity, respectively.

systematic uncertainties treatment, therefore the most important sources only are mentioned. They include b-tagging uncertainty, renormalization and factorization scale $Q^{2}$ for $\mathrm{W}$ boson production, and jet energy scale (JES). The scale factors on the b-tagging and mistagging efficiencies are varied independently within uncertainties of their data-driven estimation [3]. Simulated samples with varied $Q^{2}$ scale (half and double the nominal value) are compared to estimate the impact of the choice of renormalization and factorization scale. To account for JES uncertainties 4-momenta of all jets are simultaneously scaled up and down within the uncertainty on the data-driven jet energy corrections [4]. Further details on the sources of systematic uncertainty can be found in Ref. [1].

In $2 \mathrm{D}$ analysis the impact of each individual source of uncertainty is estimated with pseudoexperiments and the resulting asymmetric uncertainties are combined according to the prescription in Ref. [5]. On the contrary, BDT analysis treats the systematic uncertainties as the nuisance parameters and performs marginalization over them in a Bayesian manner.

\subsection{Results}

The measurements of the cross section are listed in Table 1. They are consistent among each other and with the Standard Model expectation.

The correlation between the two analyses is estimated with the pseudoexperiments and then the cross sections are combined with the help of BLUE technique [6]. The combined cross section is $83.6 \pm 29.8$ (stat.+syst.) \pm 3.3 (lumi.) pb, where the BDT analysis contributes with the largest weight ( $89 \%)$.

The obtained cross section can be translated into a limit for $\left|V_{t b}\right|$ element of the CKM matrix. Assuming $\left|V_{t d}\right|,\left|V_{t s}\right| \ll\left|V_{t b}\right|$ and using the prior knowledge that $0 \leq\left|V_{t b}\right|^{2} \leq 1$, the lower bound is set to $\left|V_{t b}\right|>0.62(0.68)$ from the 2D (BDT) analysis at the $95 \%$ confidence level.

\section{Analysis in tW Channel}

\subsection{Event Selection}

The signal signature in tW channel is two isolated leptons of opposite electric charge, exactly one b-jet within the acceptance, large enough $\mathbb{E}_{T}$ due to neutrinos. This results in three leptonic final states: ee, $\mathrm{e} \mu$, and $\mu \mu$ (as mentioned above $\tau$ leptons are not considered explicitly).

More specifically, an event is required to pass a dilepton trigger and have exactly two tight leptons of opposite charge satisfying $p_{T}>20 \mathrm{GeV} / c,|\eta|<2.4(2.5)$, and $I<0.15$ for a muon (electron). Additional loose muons (electrons) with $p_{T}>10(15) \mathrm{GeV} / c$, and $I<0.2$ are vetoed. 
In ee and $\mu \mu$ final states the event is rejected if the leptons' invariant mass satisfies $81<$ $m_{11}<101 \mathrm{GeV} / c^{2}$ ) or $m_{11}<20 \mathrm{GeV} / c^{2}$. The former selection reduces contribution from $\mathrm{Z}$ boson production processes, and the latter one excludes a region found to be not well described in simulation (this region also adds almost no sensitivity to the analysis). In these leptonic channels the event is additionally required to have $B_{T}>30 \mathrm{GeV}$ in order to further reduce the contribution from the Drell-Yan process. In the e $\mu$ channel instead the non-top backgrounds are further rejected by demand that the scalar sum of $p_{T}$ of the two leptons, the jet, and the $E_{T}$ is greater than $160 \mathrm{GeV} / c$.

The event is required to have exactly one jet with $p_{T}>30 \mathrm{GeV} / c$ and $|\eta|<2.4$. The selected jet must be tagged with the b-tagging algorithm exploiting properties of the reconstructed secondary vertex. Additional b-tagged jets with $p_{T}>20 \mathrm{GeV} / c$ are prohibited.

In order to suppress the $\mathrm{t} \overline{\mathrm{t}}$ background the transverse momentum of the system formed by the two leptons, the jet, and the $E_{T}$ is required to be less that $60 \mathrm{GeV} / c$.

\subsection{Data-Driven Background Estimation}

After the event selection is performed, the main backgrounds are the top pair production and the Drell-Yan process; the rest backgrounds take $\lesssim 1 \%$ of the total event yield. The two dominating processes are estimated from data.

The number of Drell-Yan events in the signal selection is estimated with the events failing the $\mathrm{Z}$ veto according to the formula

$$
N_{\mathrm{ll}, \text { out }}^{\text {estimated }}=\frac{N_{\mathrm{ll}, \mathrm{out}}^{\mathrm{MC}}}{N_{\mathrm{ll}, \text { in }}^{\mathrm{MC}}} \cdot\left(N_{\mathrm{ll}, \text { in }}^{\mathrm{observed}}-\frac{1}{2} k \cdot N_{\mathrm{e} \mu, \text { in }}^{\mathrm{observed}}\right),
$$

where $N_{\mathrm{ll} \text {, in }}^{\mathrm{MC}}\left(N_{\mathrm{ll}, \text { out }}^{\mathrm{MC}}\right)$ is the number of simulated $\mathrm{Z} / \gamma^{*} \rightarrow 1^{+} 1^{-}(1=\mathrm{e} \mu)$ events inside (outside) the $\mathrm{Z}$ mass window, $N_{\mathrm{ll}}^{\mathrm{observed}}$ in number of observed ee or $\mu \mu$ events inside the window in data, and the number $N_{\mathrm{e} \mu \text {, in }}^{\text {obsed }}$ of e $\mu$ events in data inside the window estimates the impact of non-peaking processes (mostly $\mathrm{t} \bar{t}$ and the signal) inside the $\mathrm{Z}$ window. Factor $k$ takes into account the differences in acceptance and reconstruction efficiencies for electrons and muons; it is extracted from data comparing the numbers of near-peak events in ee and $\mu \mu$ channels after the leptonic step of the event selection.

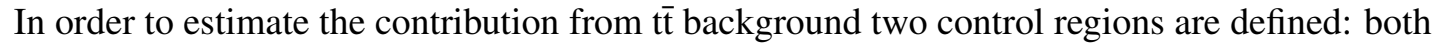
of them demand the event to have exactly two jets (meeting the same definition as in the signal event selection) and exactly one (first control region) or exactly two (second one) of them must be $b$-tagged. These regions are by far dominated by $t \bar{t}$ production. The corresponding numbers of events are used as input to the statistical procedure and constrain the process' normalization and b-tagging efficiency.

\subsection{Cross-Section Measurement and Results}

The tW channel cross-section is measured with a likelihood fit to the number of events in the signal and control regions (Fig. 4). The latter are used to constrain the $t \bar{t}$ background and the b-tagging efficiency. The systematic uncertainties are treated as the nuisance parameters in a Bayesian inference.

A detailed description of the sources of systematic uncertainties would be out of volume limitation for this article. However the most of them are the same as for the $t$ channel analysis, which 

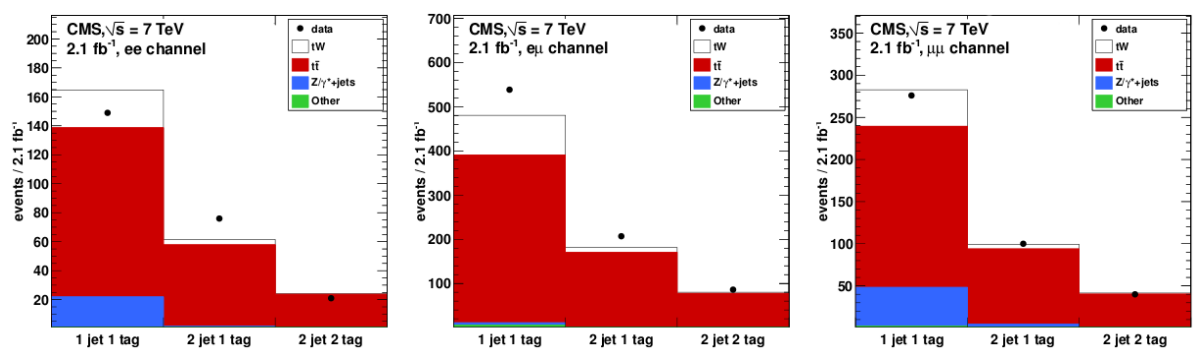

Figure 4: Event yields in the signal and $\bar{t} \bar{t}$ control regions in three leptonic final states. Drell-Yan process scaled to the data-driven estimate, $t \bar{t}$ production process scaled to the result of the fit.

were partly described in Sec. 2.5. One notable exception specific for the tW channel is the ambiguity in the signal definition due to the interference with the tit process at NLO. The signal is simulated in two different schemes, "diagram removal" (DR) and "diagram subtraction" (DS) (see Refs. in Ref. [2]). The DR sample is used as the default in the analysis whereas the difference from DS sample is treated as an additional systematic uncertainty. The details can be found in Ref. [2].

The measured cross-section is $22_{-7}^{+9} \mathrm{pb}$ (stat.+syst.).

\section{Conclusions}

Two channels, $\mathrm{t}$ and $\mathrm{tW}$, of single top quark production have been studied at CMS experiment. In the $t$ channel two complementary analyses have been performed: one exploited two angular variables having characteristic distributions for the signal and another one used a multivariate technique relaying on the good agreement between the data and simulation. Each of the analyses (re)found an evidence for the $t$ channel production. They were combined and the cross-section was found to be $83.6 \pm 29.8$ (stat.+syst.) \pm 3.3 (lumi.) pb. In the tW channel cross-section $22_{-7}^{+9} \mathrm{pb}$ (stat.+syst.) was measured with a counting experiment. All the results are in good agreement with the Standard Model expectations.

\section{References}

[1] CMS Collaboration, Measurement of the t-Channel Single Top Quark Production Cross Section in pp Collisions at $\sqrt{s}=7 \mathrm{TeV}$, Phys. Rev. Lett. 107091802 [arXiv:1106.3052].

[2] CMS Collaboration, Search for single top $t W$ associated production in the dilepton decay channel in pp collisions at $\sqrt{s}=7 \mathrm{TeV}$, CMS PAS TOP-11-022.

[3] CMS Collaboration, Commissioning of b-jet identification with pp collisions at $\sqrt{s}=7 \mathrm{TeV}$, CMS PAS BTV-10-001.

[4] CMS Collaboration, Determination of the Jet Energy Scale in CMS with pp Collisions at $\sqrt{s}=7 \mathrm{TeV}$, CMS PAS JME-10-010.

[5] R. Barlow, Asymmetric Systematic Errors [arXiv: 0306138].

[6] L. Lyons, D. Gibaut, P. Clifford, How to combine correlated estimates of a single physical quantity, Nucl. Instrum. Meth. A270 110. 